\title{
Secure Medical Data Transmission in Body Area Sensor Networks Using Dynamic Biometrics and Steganography
}

\author{
R. Rekha, T. Gayathri Mathambigai, and Dr.R. Vidhyapriya
}

\begin{abstract}
In this paper, we have used ECG signal for secure key exchange between two nodes of the same Body Sensor Network (BSN). InterPulsed Interval (IPI) of the ECG signal is considered as a biometric characteristic for key generation because of its high randomness, time variance properties and also to avoid interference between the other BSNs. The body sensor node to base station (PDA or Laptop) communication has been secured using the symmetric cryptographic algorithm AES and integrity is checked by using the Message Authentication Code (MAC). Steganography is used for securing the data transfer between base station and medical server. In this work, we have embedded the medical data (Blood Pressure) within the patients' facial image. The face image is selected as a cover image in order to provide authentication. The pixel positions for embedding the medical data are determined by fixing a threshold level (120-126). This threshold value is chosen so as to have high random distribution throughout the image. The results have been shown using MATLAB 7.8.0.
\end{abstract}

Keywords--- ECG Signal, InterPulsed Interval, Medical Data, Steganography

\section{INTRODUCTION}

$\mathrm{W}$ IRELESS sensor networks (WSN) are the collection of nodes that are organized in an ad-hoc manner. Each node will have the ability to compute, communicate and have a battery as a rechargeable source. WSN is used for various applications such as health monitoring, environmental sensing, area monitoring, air pollution monitoring etc. We have considered the health monitoring application of WSN and the security related issues are analyzed. Wireless Body Area Sensor Networks (WBASN) are the networks of medical sensors in or around the human body to monitor their vital signs. Biosensors are used to analyze the biological samples to know about their structure and function.

WBASN can use the Wireless Personal Area Networks (WPAN) to communicate over the shorter ranges i.e., from the nodes of the BSN to the base station. The base station is the mobile or Personal Digital Assistant. IEEE classifies IEEE 802.15.6 for the BSN with the scope of "short range, wireless

Rekha, Assistant Professor, Department of Information Technology, PSG College of Technology, Coimbatore, India, E-mail:rekha.psgtech@gmail.com GayathriMathambigai, PG Student, Department of Information Technology, PSG College of Technology, Coimbatore, India, Email:tgayathri12@gmail.com

Vidhyapriya, Associate Professor, Department of Information Technology, PSG College of Technology, Coimbatore, India, Email:rvidhyapriya@gmail.com communication on or inside the human body" [1].

The health monitoring is very important to lead a quality life. BSN is mainly used for the patients who need continuous monitoring to diagnose about the severity of the diseases, known as Chronic Disease monitoring. Also it may be used to monitor the fitness and wellness of the patients. For example, an athlete may check his/her heart beat or blood pressure during running. Healthy patients can also use this for detecting any type of diseases in their body. BSN can be used for cardiovascular diseases, cancer detection, depression, elderly people monitoring, and glucose level monitoring. The security is the major concern in the BSN since the medical information of the patients is sent through the wireless link. Some of the security protocols for the BSN are TinySec, MiniSec, SPINS and ECC.

WPAN like Bluetooth, Zigbee can be used for transmitting the information from node to base station. Zigbee, IEEE 802.15.4 is less expensive and consumes less power than Bluetooth. Zigbee supports all types of healthcare monitoring devices like ECG sensors, Glucose Meter and Pulse Oximeter [2]. It supports for point-of-care medical device communication. Also, the Zigbee does not affect the human since its duty cycles are thousand times less than Bluetooth. Zigbee also have some disadvantages like high maintenance cost and low data rate. It is an emerging field so the improvements in Zigbee can make it to work in a better way.

We have discussed briefly about the BSN architecture, ensuring security in the inter-BSN, intra-BSN and from the base station to external server in the later sections.

\section{BODY AREA SENSOR NETWORKS}

Body Area Sensor Networks are also termed as Body Area Networks (BAN), Body Sensor Networks (BSN) and Wireless Body Area Sensor Networks (WBASN). Each WBASN will have the base station to which it has to send the data. The vital signs are measured by the biosensors placed in the human body and it has to be transmitted to the base station. The PDA or mobile acts as a base station. The base station will send the medical data to the medical server.

In Fig. 1, the biosensors placed in the human body senses the data and it sends to the base station using the IEEE 802.15.4 standard, Zigbee. The base station transmits the medical data to the hospital server and it is stored in a database for future reference of the patients' family members or doctors. The base station transmits through the home gateway. It transmit the vital signals through the external gateway during the emergency conditions i.e., abnormal range 
of data. The doctor or caregiver can view the details of the patients' signals and they can prescribe the medicine to the patients. The three levels of communication are shown in Fig. 1. The levels are,

- Communication between the two nodes of same BSN (Intra-BSN Communication)

- Communication between the node and base station (Inter-BSN Communication)

- Communication between the base station and medical server (External Network)

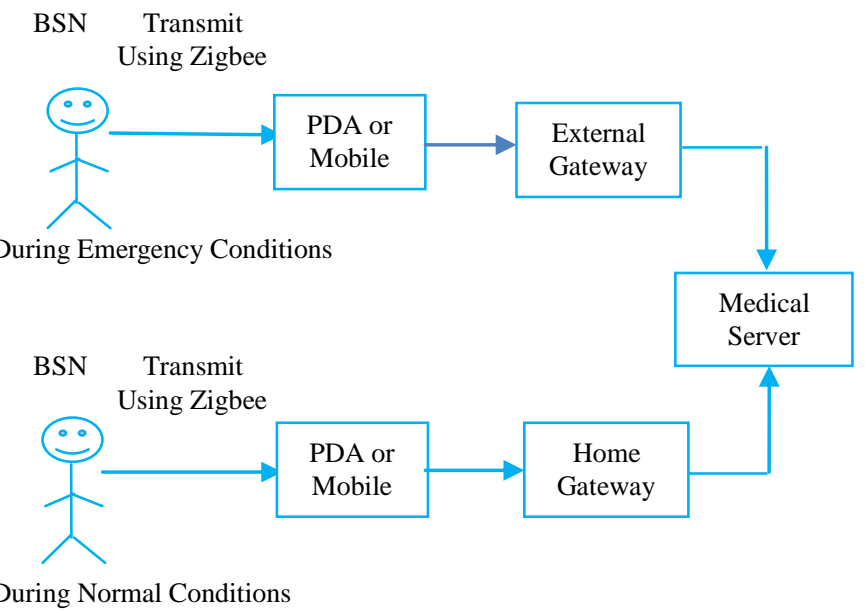

Figure 1: Body Sensor Network Architecture

The intruder may access the medical data and attempt to change the medical information which may cause harm to the patients' health. Also they may try to change the drugs prescribed by the doctor. According to Health Insurance Portability and Accountability Act (HIPAA), the patients' personal information and medical data should be kept confidential. Thus the security is the major concern in WBASN. Eavesdropping has to be avoided and the integrity of the data should be maintained. Since the biosensors are placed in the WBASN, it has many constraints than the normal sensor networks. Due to its miniature nature, it has some constraints like low power, limited memory, low communication rate and low computational complexity.

There are some specific requirements for implementing the BSN [9]. The requirements include

- Placement of sensors on the body at a 2-3 meters distance since it can communicate only shorter ranges,

- Interference between a node of BSN with the node of different BSN has to be avoided,

- Should allow to place any type of sensor in the BSN

- Should be taken care of sending the information to the destination without loss of path since it is the major issue in the wireless sensor networks.

Among the three levels of communication, first the InterBSN communication will take place and the BSN share a secret key with the base station. Then the Intra-BSN communication may take place. The nodes of the BSN tend to communicate with another node for transmitting the data. We need to ensure that the requesting nodes belong to the same
BSN. The nodes of different BSN should not communicate with each other to avoid the interference. It causes damage to the patients' health.

The security requirements of BSN are integrity, data freshness, confidentiality, integrity, availability and authenticity. The security methods available for secure communication are key distribution, pseudorandom numbers and pre-deployment of keys in the sensors [10].

Key distribution uses cryptographic algorithms for exchanging the keys between the two nodes of BSN. Due to the constraints mentioned above, asymmetric cryptographic algorithms cannot be used because it has larger computations. Pseudorandom numbers can be used for sharing the secret key but the same sequence cannot be generated at the other side due to different hardware. So it cannot be used in the BSN. Pre-deployment of keys is not an efficient solution since it creates issue during the addition and removal of sensor nodes. If the new sensor nodes are added or deleted then it is not possible to change the key in the already implanted sensors. Hence it is not a suitable method for the security purpose.

Thus the biometrics is the possible solution for sharing the secret key in a secure manner. Biometrics is used to identify the individuals uniquely using physiological characteristics and behavioral characteristics of the person. The physiological biometrics include fingerprint recognition, face recognition, voice recognition, iris recognition etc. The behavioral biometrics include signature recognition, keystroke dynamics etc. These are the conventional biometrics and it will not be suitable to provide security in the BSN because it is time invariant. It is also proved that the physiological signals of the persons can be used as a biometric trait and it is known as novel biometrics. The novel biometrics is time variant, universal, recoverable and has random. The physiological signals like ECG, Phonocardiogram (PCG), blood pressure, blood glucose level can be used as a biometric trait. It satisfies the requirements of a good cryptographic key i.e., it is having high randomness and varies according to the time.

The first level of the BSN is secured by using the novel biometrics and also the interference is avoided. The second level of the BSN is the communication between the node and base station which has been secured by using the cryptographic and hash algorithm. The third level of the BSN uses Steganography for sending the medical data to the external server. Steganography is an art of hiding the secret messages in the cover image for secure data transmission. This technique has been used in the BSN for securing the medical data that is transmitted through the external link.

\section{RELATED WORK}

Cherukari et al., proposed the use of novel biometrics in the BSN for the security purpose. Fuzzy Commitment scheme has been used for transmitting the key in a secure manner. The secret key is committed using the physiological signal and it is decommitted in the other side. Consider $\mathrm{c}$ is the key and the generated physiological signal is $\mathrm{x}$. c will be committed with $\mathrm{x}$. At the other side, consider the generated signal is $\mathrm{y}$. It uses $\mathrm{y}$ to decommit the $\mathrm{c}$ from the committed data.RC5 algorithm 
was used. Due to the larger computation, bandwidth increased and it leads more power consumption. Cherukari et al., mentioned that the timing information of the heart beat cannot be used as a biometric trait since it have less entropy [3].

C.Y. Poon et al., proved that the timing information of heart beat can be used as a biometric trait. ECG signal has been used as a biometric trait. The features like P, PQR, QT, QS can be derived from the ECG signal and it can be used for securing the key distribution. Synchronization of different problems is the main problem in this method. Also it is vulnerable to some attacks like Ultra Wide Band and Radar. Fuzzy Commitment scheme was used [5].

S.M.K. Raazi et al., proposed a key distribution scheme, BARI. Here they have considered the problem of synchronization and it had been overcome by the key refreshment module. It depicts the turn of each node for its refreshment. Three secret keys such as communication key, administrative key and basic key are used. The base station issues the new key refreshment schedule periodically. The main drawback in this method is securing the keys are not taken into consideration [7].

Fen Miao et al., proposed a method to overcome the drawbacks faced by the S.M.K. Razzi et al. Fen Miao et al., has used the cryptographic algorithms to encrypt the biometric data. AES algorithm has been used as a stream cipher in this method by feeding back the key stream. The performance of AES and RC4 algorithm has been analyzed and they suggested that AES algorithm is better when compared to RC4 in terms of memory space used, throughput. Fuzzy Vault scheme has been used [8].

F. Sufi et al., developed a novel encryption technique, a chaos based encryption. It is used for preventing unauthorized access of ECG signal during the Inter-BSN communication. The chaos key is derived from the true random numbers. The chaos key issues the true random numbers. Its efficiency is mainly dependent on the initial conditions of the server. Initial conditions are known only by the server since it is more sensitive. Diffie Hellman key exchange algorithm has been used for exchanging the chaos key between the base station and node [11].

Jun $\mathrm{Wu}$ and Shigeru Shimamoto proposed an energy efficient data secrecy scheme for the secure communication. They proposed a threshold selective encryption technique for reducing the power consumption. Selection pattern transmission technique was adopted and this method is more efficient when compared to the previous techniques [12].

\section{INTER-BSN COMMUNICATION}

The node and base station has to transmit the secret key in a secure manner. The hash algorithm and cryptographic algorithm can be used. In the proposed scheme, MD5 and DES algorithm have been used. Hash based Message Authentication Code (HMAC) can be used for calculating the Message Authentication Code (MAC) of the message. It is used to ensure the integrity of data. The steps for secure communication are shown below.

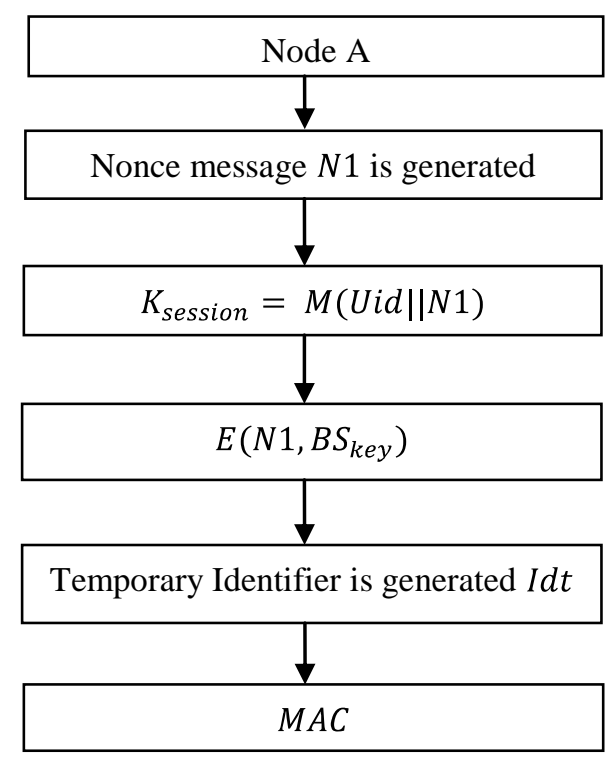

The node A will send the $I d t, E\left(N 1, B S_{\text {pubkey }}\right), M A C$ will be sent to the Base Station. The encryption is done by using the DES algorithm. The base station will perform the following steps.

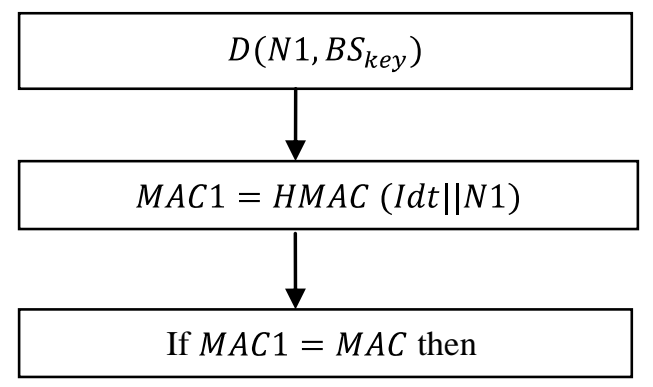

The base station will generate a Counter, used to avoid the replay attacks. It will send EKsession(N2, Counter) where $N 2$ is a nonce message. Thus the key has been shared in a secure manner.

\section{INTRA-BSN COMMUNICATION}

After sharing the secret key between node and base station, intra-BSN Communication can be established. The ECG signal is used as a biometric trait to secure the intra BSN communication. As discussed earlier, the three levels in the BSN has to be secured. The image of a biosensor to record the ECG signal is shown in Fig 2.

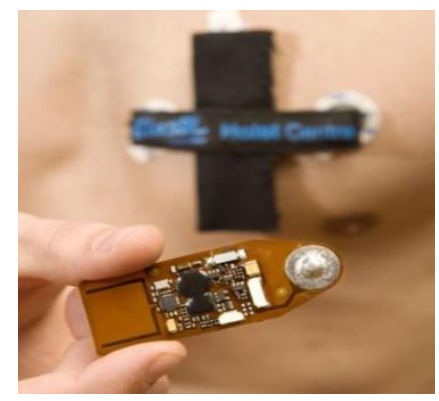

Figure 2: Wireless Biosensor to Measure ECG Signal 
The InterPulsed Interval (IPI) of the ECG signal is considered as a biometric trait in this system. The IPI is the time interval between the RR peaks in the ECG signal. The main process is generating the biokey from the ECG signal.

The biokey can be formed by using the IPI of the ECG signal. The steps for generating the biokey are shown below.

1. Generating the ECG signal in the node.

2. Sampling the ECG signal at the rate of $1000 \mathrm{~Hz}$.

3. Finding the maximum amplitude in the ECG signal.

4. Detecting the maximum peaks greater than half of the amplitude.

5. Calculating the RR interval for the detected peaks.

6. Converting into binary sequence.

7. Biokey of 128 bits is generated.

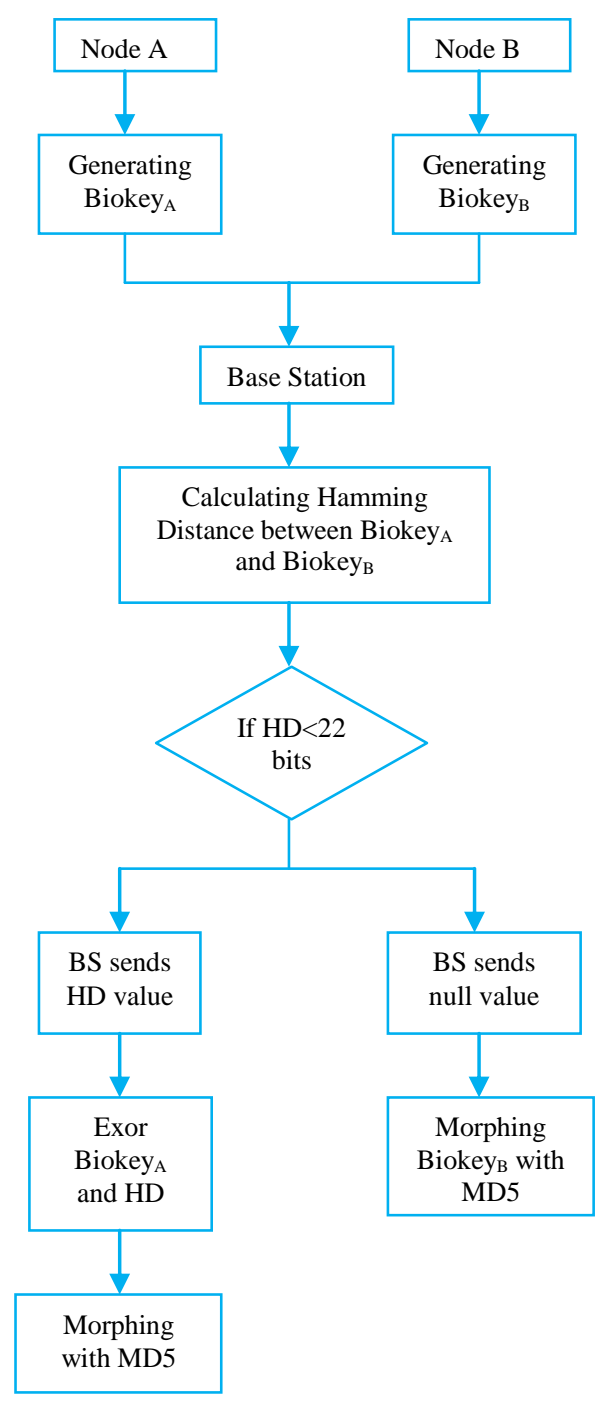

Figure 3: Secure Communication between Two Nodes in BSN

The ECG signal is measured in the respective node and the signal is sampled using the sampling frequency of $1000 \mathrm{~Hz}$. Then the maximum amplitude of the ECG signal is calculated. The R peaks will lie only on more than half of the amplitude of the signal. Hence the peaks greater than half of the amplitude has been detected. The difference between the $\mathrm{R}$ peaks of the ECG signal has been calculated. It is known as InterPulsed Interval (IPI). Then it is converted into a binary sequence to form the 128 bit biokey. After generating the biokey, the communication between the two nodes in the BSN has to be secured. The steps for secure communication between two nodes have been shown in Fig 3 .

Consider Node A needs to communicate with the Node B. Node A will measure the ECG signal and the Biokey $_{A}$ will be generated. It will send $E K_{\text {session }}\left(\right.$ Biokey $\left._{A}\right)$ to the base station. The Node B will also measure the ECG signal and generate its biometric trait Biokey $_{B}$. It will also send its encrypted biokey $E K_{\text {session }}\left(\right.$ Biokey $\left._{B}\right)$ to the base station. The base station will decrypt with the $K_{\text {session }}$ and compute the hamming distance between the Biokey $_{A}$ and Biokey $_{B}$. S.D.Bao et al., proved that the hamming distance of Heart Rate Variability (HRV) of two identical person will be less than 22 bits and for the different person it will result in the approximately 80 bits or higher difference. If the Hamming Distance (HD) between them is less than 22 bits, then the nodes are belonged to the same WBSN [4][6].

The base station will send the value of HD to the node A and sends the NULL value to the Node B. The Node A will exor the HD value and its Biokey $_{A}$. Then it is morphed with the MD5 algorithm. The Node B will exor the NULL value and its biokey. Hence the morphing has to be done for the Biokey $_{B}$. If it is approximately greater than 80 bits then it does not belong to the same WBASN and it is discarded. Thus the key has been exchanged securely between the two nodes in the WBASN.

\section{Secure Data Transmission Between Two Nodes}

The secret key has been shared securely between two nodes. Now, the two nodes of same BSN will exchange some medical information like blood pressure, temperature etc. The blood pressure is taken as a data for the experiment. It has been collected from the net in www.physionet.org from the Fantasia Database. When Node A is sending data to Node B, the data can be secured by performing scrambling operation using the random number generator. The seed can be taken from the secret key which is shared between the two nodes. 10 bits can be selected randomly from the secret key at Node A as a seed and it can be sent to Node B by encrypting the values with the AES algorithm. The 128 bits of the shared secret key can be taken as a key for AES encryption. The DCT coefficients are taken for the scrambled data and it is sent to the Node B of same BSN. At the receiver side, Node B will decrypt with AES algorithm to retrieve the seed value. The inverse DCT coefficients are taken and random number will be generated using the seed value. The descrambling can be done to retrieve the data at Node B.

\section{COMMUNICATION FROM BASE STATION TO MEDICAL SERVER}

The node will send the medical data to the base station. The base station sends the data to the medical server which is an external network. The steganographic algorithms can be used to secure the medical data between them. LSB based 
Steganography [16] and DCT based Steganography [13] [14] [15] can be used to hide the secret message (medical data) in the cover image.

The gray scale image is considered as the cover image. The grayscale pixel values ranges from $0-255$. Most of the pixels will be in the range from 100-200. Due to this reason, we have fixed a threshold value of range 120-126 so as to embed the medical data randomly in the cover image. Secret message is embedded into LSB of the randomly selected pixels.

The steps involved in the embedding process are,

1. Convert the Cover image into a gray scale image

2. Convert the data i.e., secret message into a binary number and embed into the LSB of the selected pixel positions.

3. The cover image with the secret message has been sent to the medical server. Thus the stego image is obtained.

The steps involved in the retrieval process of secret message at the medical server are given below.

1. Read the received stego image

2. Select the positions which are in between the threshold value

3. Retrieve the LSB of the positions and convert it into decimal value

4. Thus the secret message has been retrieved

Thus the medical data has been securely transmitted to the medical server from the base station.

\section{EXPERIMENTAL RESULTS}

Two sets of ECG signal have been collected for six individuals at a regular interval of five minutes. We have considered a Body Sensor Network which consists of Node A, Node B and a base Station.

Initially the nodes will share a secret key with the base station. The secret key can be used to exchange the information between the nodes and base station. It can be generated by using the nonce message (a random number) and the Unique Identifier of the particular node. The steps for secure inter-BSN communication have been explained in Section IV.

Consider Node A needs to communicate with the base station. The information sent from Node A to base station is shown in Fig. 4. The encryption is done by using DES algorithm.

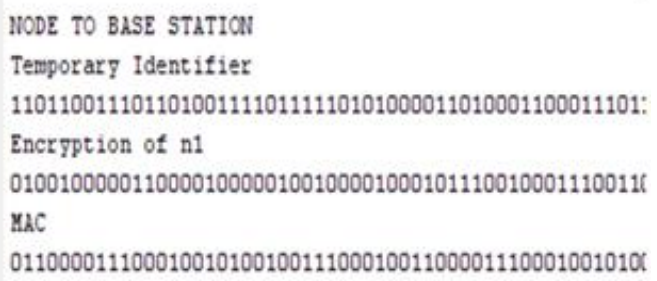

Figure 4: Node A to Base Station

The Base Station will receive the information from Node $\mathrm{A}$ and derives the nonce message $N 1$ by decrypting with its key. Then it calculates the $M A C$ and checks with the $M A C$ received from the node A. If both MAC are equal, then base station will send the counter value which is used to avoid the replay attacks.

The base station to Node A communication is shown in Fig. 5.

\section{BASE STATION TO NODE \\ Encrgption of Nonce N2 1011001101101111110110010010100110110011011011111101 Counter 1001011100010101011110100110111111001000111001001011:}

Figure 5: Base Station to Node A

The shared secret key between node A and base station is shown in Fig. 6.

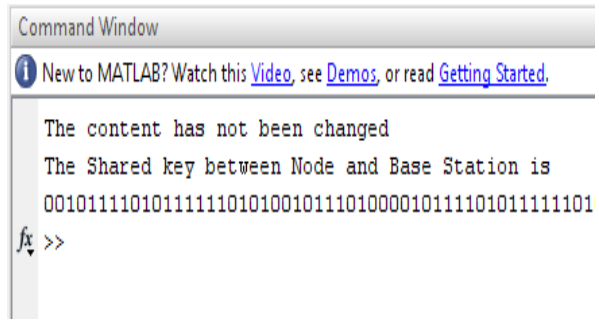

Figure 6: Secret Key between Node A and Base Station

Assume that node $\mathrm{A}$ of a person $\mathrm{X}$ has to communicate with node B of the same person. The ECG signal of a person $\mathrm{X}$ at the node $\mathrm{A}$ is generated and it is sampled at the rate of $1000 \mathrm{~Hz}$. The detected R peaks are plotted on the ECG signal of Node A (Person X) and it is shown in Fig 7.

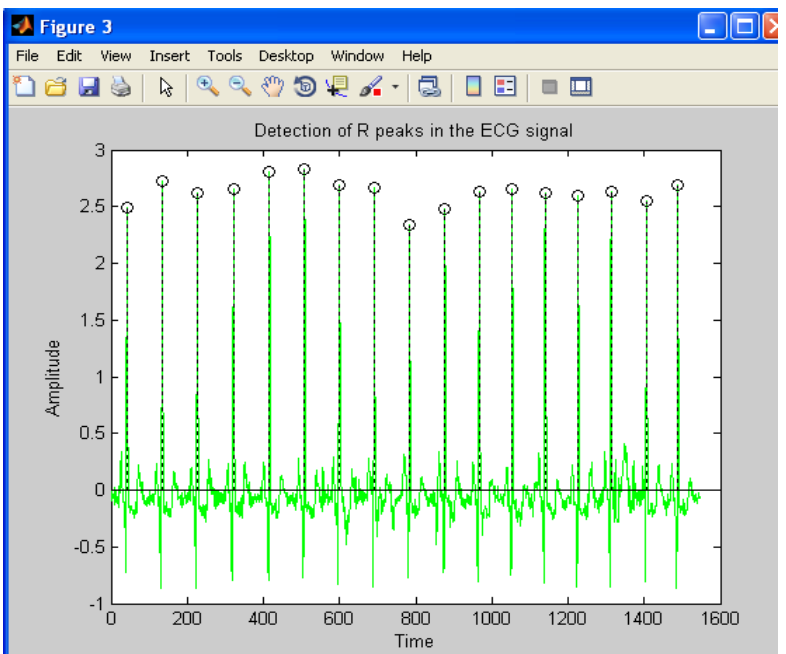

Figure 7: Detected R Peaks at Node A of Person X

The 128 bit length biokey of Node A has been generated (Person $\mathrm{X}$ ) and it is shown in Fig. 8 .

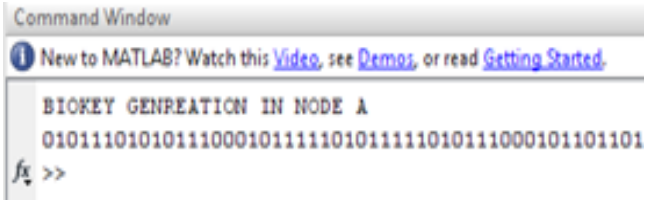

Figure 8: Biokey Generation at Node A of Person X 
At Node B, the ECG signal is generated and the detected R peaks are plotted. The biokey has been generated for Node B (Person X). The hamming distance between the biokey of Node A and Node B is calculated to know whether it belongs to same BSN or not. The obtained hamming distance for both the nodes is 16 and it is shown in Fig. 9. Thus it belongs to the same BSN.

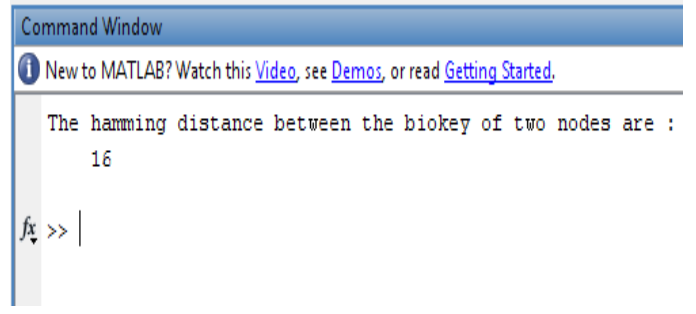

Figure 9: Hamming Distance between Node A and Node B of Person X

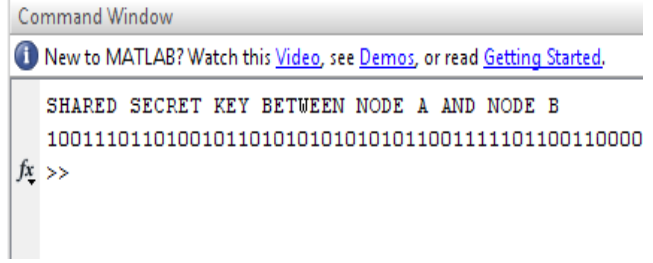

Figure 10: Shared Secret Key between Node A and Node B

MD5 algorithm has been used for morphing. The secret key is shared between two nodes A and B in a secure manner and it is shown in Fig. 10.

Consider if a medical data has to be sent to node B from node A. Here blood pressure is taken as a medical data. Node A will scramble the data and it is sent to node B. The scrambled medical data is shown in Fig. 11.

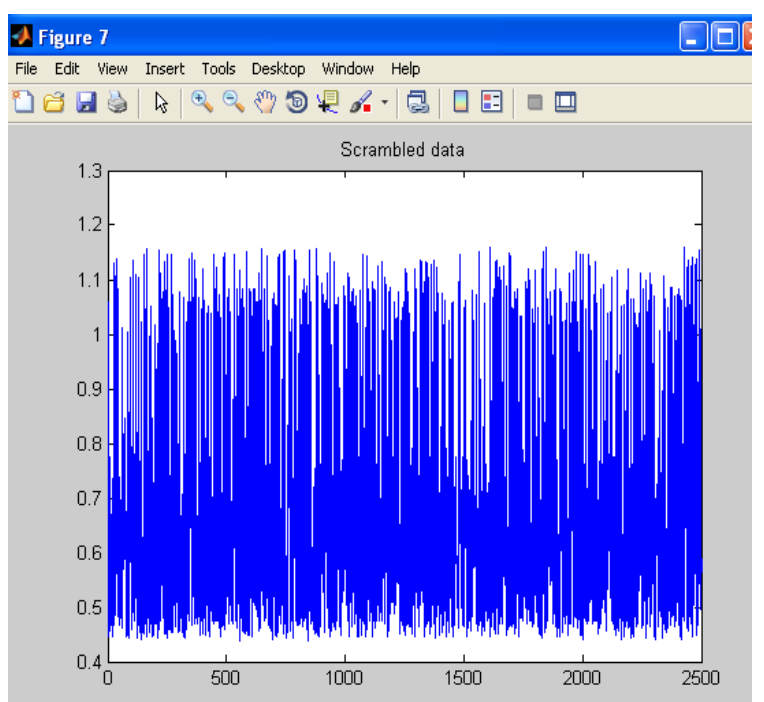

Figure 11: Scrambled Medical Data

The retrieved medical data at Node B is shown in Fig. 12.

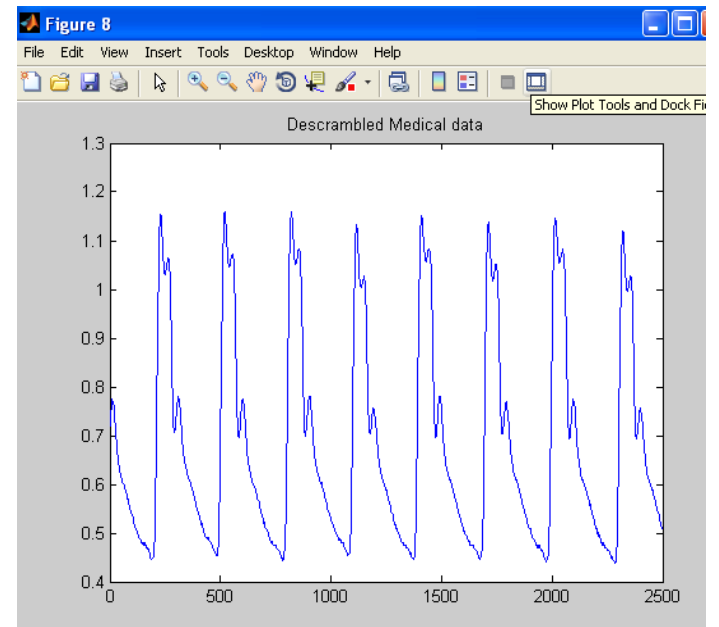

Figure 12: Descrambled Medical Data at Node B

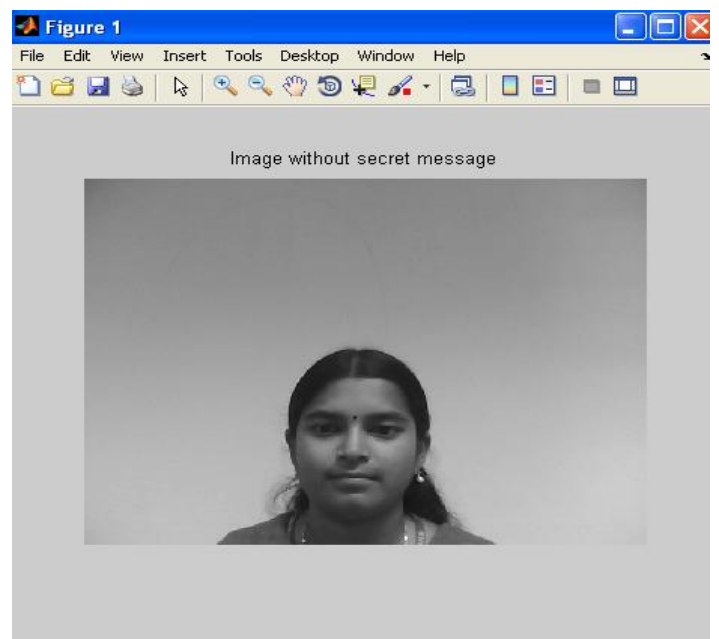

Figure 13: Original Image before Embedding

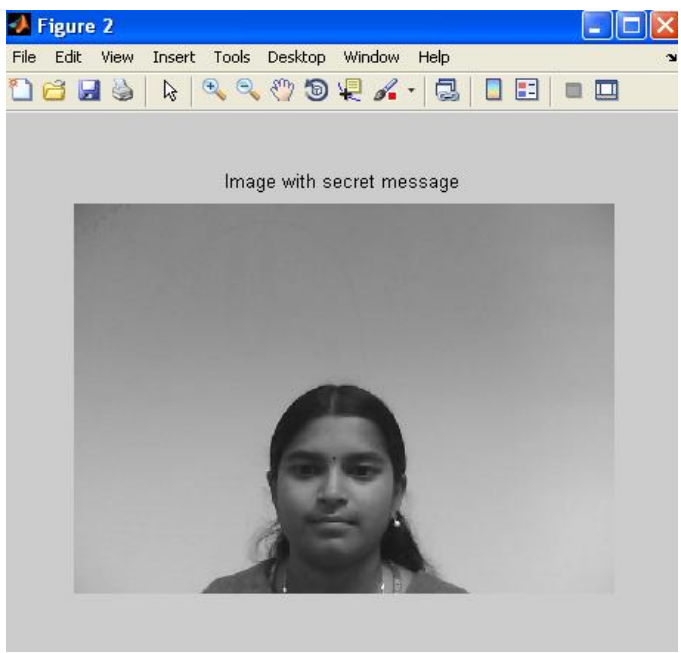

Figure 14: Stego Image after Embedding Secret Message

After transmitting the information from the Node A to base station, the base station will send to the medical server. The Steganography algorithm has been used for secure transmission. We have considered the threshold value between 120 and 126. The face image is considered as a cover image. The image before embedding process is shown in Fig. 13. 
After embedding the secret message, the stego image is shown in Fig. 14.

The retrieved medical data at the medical server is shown in Fig. 15.

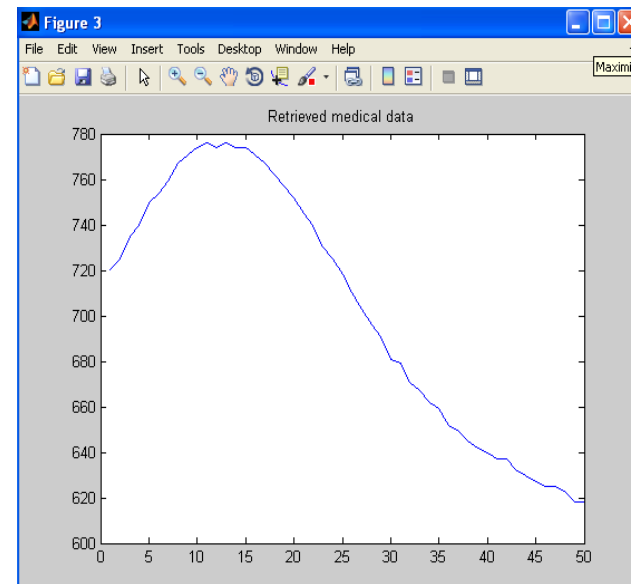

Figure 15: Medical Data at the Medical Server

Assume that node B of person Y needs to communicate with the node A of person X. The biokey of both the nodes will be generated and it will be sent to the base station. The hamming distance between them is 72 and it is shown in Fig. 16. Thus the two nodes do not belong to the same BSN. The biokey will be discarded.

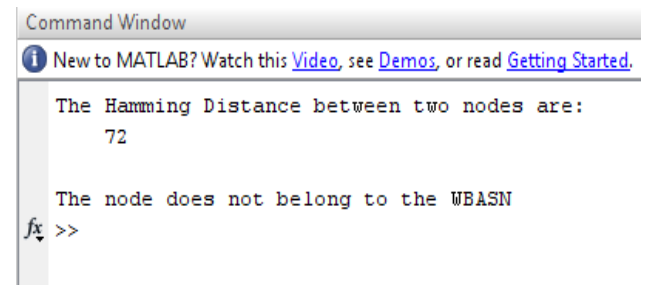

Figure 16: Mismatch between Node A and Node B of Person $\mathrm{X}$ and $\mathrm{Y}$

\section{CONCLUSION}

WBASN is one of the emerging trends in the healthcare application. It has unique importance since it is capable of monitoring the patients ubiquitously. If the security issue in the WBASN is solved, it can be used in an appropriate way. Some of the major issues in the security are considered and it has been solved in our work. Issues like interference of one node with another node, hacking of data have been solved. Data integrity is maintained by using the MAC function. The key has been securely distributed by using the inherent properties of the human body. As a future work, the performance SHA-1 can be analyzed instead of MD5 algorithm and a modified DCT based Steganography algorithm can be implemented to enhance the performance of the system and the security.

\section{REFERENCES}

[1] "IEEE 802.15.6" http://www.wisar.org/research/ieee-802156.html

[2] "Zigbee Alliance" http://www.zigbee.org

[3] Sriram Cherukuri, Krishna K Venkatasubramanian and Sandeep K S Gupta "BioSec: A Biometric Based Approach for Securing Communication in Wireless Networks of Biosensors Implanted in the
Human Body", Proc. IEEE Int'l. Conf. Parallel Processing Workshops Pp. 432-39, Oct 2003

[4] S.-D. Bao, Y.-T. Zhang, and L.-F. Shen, "Physiological signal based entity authentication for body area sensor networks and mobile healthcare systems", Proceedings of the 27th Annual International Conference of the IEEE Engineering in Medicine and Biology Society, Pp. 2455-2458, September 2005.

[5] C. Poon, Y. T. Zhang, and S. D. Bao, "A novel biometrics method to secure wireless body area sensor networks for telemedicine and $\mathrm{m}$ health", IEEE Communication Mag., Vol. 44, No. 4, Pp. 73-81, April 2006.

[6] F. M. Bui and D. Hatzinakos, "Biometric methods for secure communications in body sensor networks: resource efficient key management and signal-level data scrambling," EURASIP Journal of Advances in Signal Processing, Vol. 8, No. 2, Pp. 1-16, 2008.

[7] Syed Muhammad Khaliq-ur-Rahman Raazi, Sungyoung Lee, YoungKoo Lee and Heejo Lee, "BAR I: A Distributed Key Management Approach for Wireless Body Area Networks", IEEE Conference 2009.

[8] Fen Miao, Lei Jiang, Ye Li and Yuan-Ting Zhang, "A Novel Biometrics Based Security Solution for Body Sensor Networks, IEEE conference 2009

[9] Mohammed MANA, Mohammed FEHAM and Boucif AMAR BENSABER, "SEKES (Secure and Efficient Key Exchange Scheme for wireless Body Area Network)", IJCSNS International Journal of Computer Science and Network Security, Vol. 9, No. 11, November 2009.

[10] Shahnaz Saleem, Sana Ullah and Hyeong Seon Yoo, "On the Security Issues in Wireless Body Area Networks ", International Journal of Digital Content Technology and its Applications, Vol. 3, No. 3, September 2009.

[11] F. Sufi, F. Han, I. Khalil and J. Hu, "A chaos-based encryption technique to protect ECG packets for time critical telecardiology applications", Security and Communication Networks, 2010.

[12] Jun Wu and Shigeru Shimamoto, "An Energy Efficient Data Secrecy Scheme for Wireless Body Area Sensor Networks", Computer Science \& Engineering: An International Journal (CSEIJ), Vol. 1, No. 2, June 2011.

[13] Ajit Danti and Preethi Acharya "Randomized Embedding Scheme Based on DCT Coefficients for Image Steganography", IJCA Special Issue on Recent Trends in Image Processing and Pattern Recognition, 2010.

[14] Dr. Ekta Walia, Payal Jain and Navdeep," An Analysis of LSB \& DCT based Steganography", Global Journal of C omputer Science and Technology, Vol. 10, No. 1, April 2010.

[15] K.B. Shiva Kumar , K. B. Raja, R.K. Chhotaray, and Sabyasachi Pattanaik, "Bit Length Replacement Steganography Based on DCT Coefficients", Vol. 2, No. 8,Pp. 3561-3570, 2010.

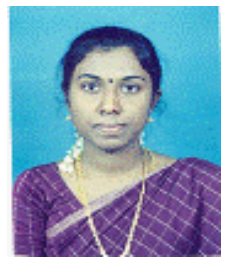

Rekha has received B.E (ECE) from PSNA College of Engineering and Technology, Dindigul, India in 2003 and M.E Communication Engineering from Periyar Maniammai College of Technogy for Women, Thanjavur, India in 2009. Her areas of interests are Wireless Networks and Communication Engineering. She has published her research works in 4 International Conferences.

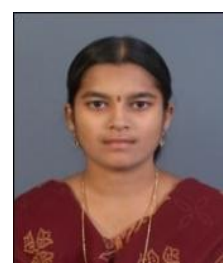

GayathriMathambigai has received B.E (CSE) from Vivekanandha Institute of Engineering and Technology for Women, Tiruchengode, India in 2010. Her areas of interests are Biometrics, Wireless Sensor Networks. She has published in 4 International Conferences.

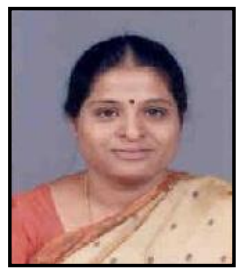

Dr.R. Vidhyapriya has received B.E (ECE), M.E (Applied Electronics) and Ph.D from PSG College of Technology, Coimbatore, India in 1992, 1999 and 2009 respectively. Her major field of study is Electrical and Electronics Engineering. Her areas of interests are wireless networks and Data Communication. She has published 6 International Journals and 7 International Conferences. 